\title{
The influence of a DC electric field on the von Kármán vortex street in the wake of a confined cylinder
}

\author{
Mathias Scholz ${ }^{1}$, Steffen Hardt ${ }^{2}$ \& Dominik P.J. Barz ${ }^{1,2, *}$ \\ ${ }^{1}$ Department of Chemical Engineering, Queen's University, \\ Kingston ON K7L 3N6, Canada \\ 2 Institute for Nano- and Microfluidics, Technische Universität Darmstadt, \\ D-64287 Darmstadt, Germany, \\ *E-mail: dominik.barz@queensu.ca
}

\section{Introduction}

The von Kármán vortex street is a flow instability that can be observed in the wake of a bluff body if a critical Reynolds number is exceeded. The present report is concerned with the numerical simulation of the von Kármán vortex street induced by a pressure-driven flow around a confined cylinder that is subjected to a DC electric field. We note two distinctions to the classical von Kármán vortex street for such an arrangement. The confinement of the flow field results in an additional shear in the incoming velocity profile and the channel walls and also induces shear in the vicinity of the boundaries. Additionally, the confinement is a natural barrier for the propagation of the vortices. The presence of the electric field induces a Coulomb force in the electrical double layer that is located at the interface of the cylinder and the liquid. This Coulomb force induces a small "electrokinetic" flow in the vicinity of the cylinder surface contrary to a sole pressure-driven case. Review on electrical double layers and electrokinetic phenomena is available in Ref. [1]. We perform numerical simulations in the Laminar Vortex Shedding Regime to investigate the influence of such a DC field on the settling time and the lift coefficient of the von Kármán vortex street.

\section{Simulation Methodology}

The derivation and details of the mathematical model that we use to perform the numerical simulations are given in more detail in Ref. [2]. All following mathematical formulations are related to a local wall-normal and wall-tangential coordinate system $(x, y)^{T}$ with the origin at the channel wall. The two-dimensional (2D) case is a reasonable approximation for cylinder Reynolds numbers lower than 180 [2]. The governing equation of the (non-dimensional) electrical potential $\varphi$ is the Laplace equation

$$
\Delta \varphi=0
$$

with the corresponding boundary conditions $\partial_{y} \varphi=0$ at all channel walls and the in- and outlet. At the electrodes, Dirichlet conditions $\varphi=$ const are used to induce the electric field.

The governing equations of the flow field are the standard (non-dimensional) Navier-Stokes equation:

$$
\begin{aligned}
\nabla \cdot \mathbf{u} & =0, \\
R e_{c h}\left(\partial_{t} \mathbf{u}+(\mathbf{u} \cdot \nabla) \mathbf{u}\right)-\Delta \mathbf{u}+\nabla p & =0 .
\end{aligned}
$$

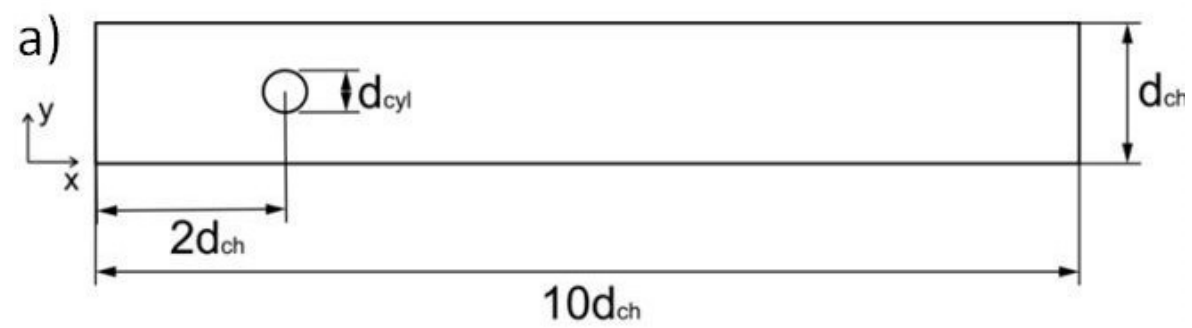

Figure 1: Schematic of the simulation domain. 

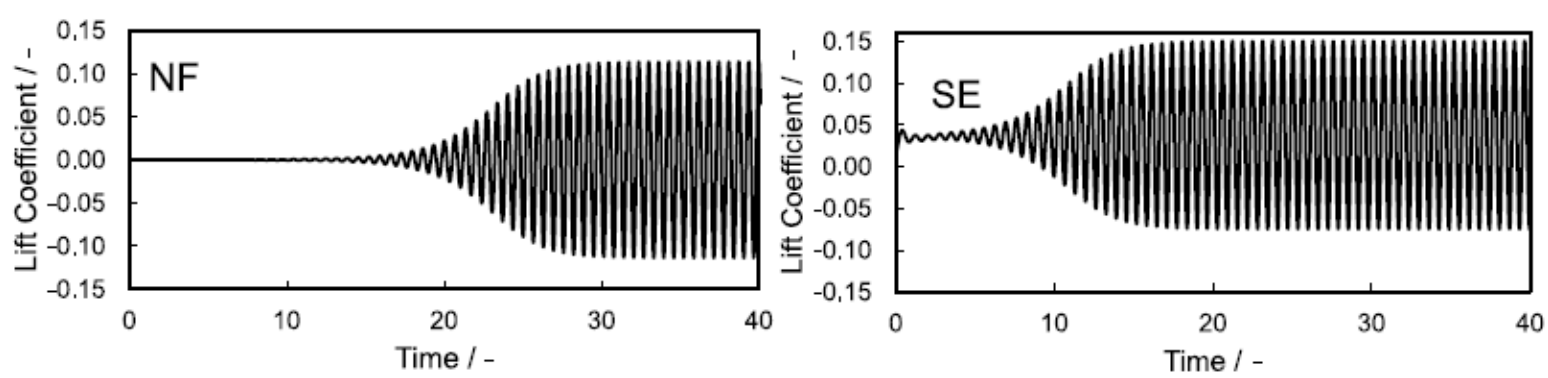

Figure 2: Evolution of the cylinder lift coefficient over time for a pressure-driven flow with $R e=60$ and $\varepsilon=0.1$ : No electric field (NF) and electric field with $\Pi=0.014$ (SE).

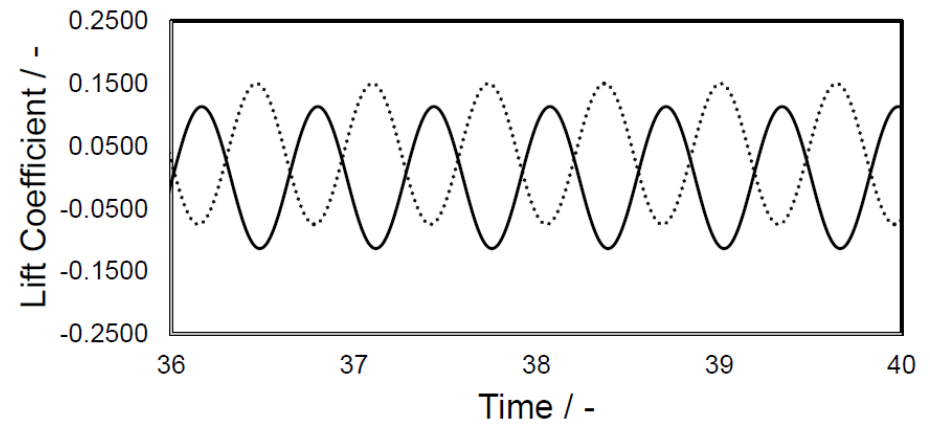

Figure 3: Fully developed lift coefficient over time for a pressure-driven flow around the cylinder with $R e=60$ and $\varepsilon=0.1$ : No electric field (solid line) and electric field with $\Pi=0.014$ (dashed line).

were $R e_{c h}=u_{0} d_{c h} / \nu$ is the channel Reynolds number. Conversion from channel to cylinder Reynolds number using the blockage ratio $\varepsilon=d_{c y l} / d_{c h}$ is straightforward; i.e., $R e=R e_{c h} \varepsilon$. The influence of the electric field on the flow is solely captured by the boundary conditions. We have $\mathbf{u} \simeq-\Pi\left(\partial_{x} \varphi, 0\right)^{T}, p \simeq \operatorname{Const}(x, 0)$ on the channel walls, along with a parabolic inlet profile and a non-viscous stress outlet boundary condition. The dimensionless group $\Pi$ can be interpreted as ratio of electric to viscous forces. Figure 1 gives a schematic of the simulation domain.

\section{Results}

Figure 2 shows the evolution of the lift coefficient for a pressure driven flow at $R e=60$ and a confinement of $\varepsilon=0.1$ without electric field (NF) and with electric field (SE) where the ratio of electric to viscous forces is with $\Pi=0.014$ very small. A considerable difference between the oscillations is obvious right away. The settling time for the case without electric field is around 26.4 while it takes only around 13.9 when the electric field acts on the flow. We also realize that the origin of the oscillation, despite the very small perturbation, is shifted from zero (no electric field) to 0.038 which is quite significant considering the small amplitude of the lift coefficient. Figure 3 gives insight into the steady lift coefficient oscillations over time. We find that the influence of the electric field on the amplitude is negligible. In case of no electric field, the amplitude of the pressure-driven flow with $R e=60$ is 0.113 while it is 0.120 with electric field turned on. Likewise, a Fast Fourier Transformation analysis reveals that the shedding frequency, expressed with the dimensionless Strouhal number $S t=f d / u_{0}$, is with 0.132 and 0.131 practically the same. It is noteworthy to mention that the phase shift between the lift coefficient oscillations is $\pi$; that is, the two oscillations are in anti-phase.

\section{References}

[1] A. V. Delgado, F. Gonzalez-Caballero, R. J. Hunter, L. K. Koopal, and J. Lyklema, "Measurement and interpretation of electrokinetic phenomena", J. Colloid Interface Sci. (309) 2007, 194-224.

[2] D.P.J. Barz, H.F. Zadeh, and P. Ehrhard, "Measurement and the simulation of time-dependent flow fields within an electrokinetic micromixer", J. Fluid Mech. (676) 2011, 265-293.

[3] C. Williamson, "Vortex dynamics in the cylinder wake", Ann. Rev. Fluid Mech., (28) 1996, 477-539. 